\title{
QUANTUM OBSERVABLES AND EFFECT ALGEBRAS
}

\author{
ANATOLIJ DVUREČENSKIJ ${ }^{1,2}$ \\ 1 Mathematical Institute, Slovak Academy of Sciences \\ Štefánikova 49, SK-814 73 Bratislava, Slovakia \\ ${ }^{2}$ Depart. Algebra Geom., Palacký Univer. \\ 17. listopadu 12, CZ-771 46 Olomouc, Czech Republic \\ E-mail: dvurecen@mat.savba.sk
}

\begin{abstract}
We study observables on monotone $\sigma$-complete effect algebras. We find conditions when a spectral resolution implies existence of the corresponding observable. The set of sharp elements of a monotone $\sigma$-complete homogeneous effect algebra is a monotone $\sigma$-complete subalgebra. In addition, we study compatibility in orthoalgebras.
\end{abstract}

\section{INTRODUCTION}

The notion of an observable is a basic mathematical tool for study of quantum mechanical measurements. It is well known that quantum mechanical events do not satisfy axioms of the Kolmogorov axiomatical model, see BiNe, $\mathrm{Var}$, and rather have a more general structure. Mathematical foundations of quantum mechanics use many important algebraic structures, like orthomodular lattices, orthomodular posets, or orthoalgebras. The most important ones of them are two structures connected with a Hilbert space which model the so-called Hilbert space quantum mechanics. So, if $H$ is a real, complex or quaternionic Hilbert space, let $\mathcal{L}(H)$ be the system of closed subspaces of $H$, then $\mathcal{L}(H)$ is a complete orthomodular lattice which is isomorphic to the set $\mathcal{P}(H)$ of all orthogonal projections on $H$. Observables in this structures correspond to orthogonal projector-valued measures, or equivalently, to symmetric operators. These structures describe so-called yes-no events. A more general structure is the system $\mathcal{E}(H)$ of Hermitian operators on $H$ that are between the zero operator $O$ and the identity operator $I$. It describes yesno events as well as so-called fuzzy events, and observables correspond to POVMs (positive operator valued measures). Of course, $\mathcal{P}(H) \subset \mathcal{E}(H)$.

Effect algebras have appeared in the beginning of the Nineties, see [FoBe]. They combine both sharp and unsharp modeling of quantum events. For them observables are defined as $\sigma$-homomorphisms from the Borel $\sigma$-algebra into a monotone $\sigma$-complete effect algebra. Today there is a reach literature on observables, see e.g. DvPu, JPV1, JPV2, DvKu, Dvu3, and others. Recently the so-called Olson order

\footnotetext{
${ }^{1}$ Keywords: Effect algebra, lattice effect algebra, monotone $\sigma$-complete effect algebra, observable, spectral resolution, homogeneous effect algebra, compatibility, orthoalgebra, orthoalgebraic skeleton

AMS classification: 81P15, 03G12, 03B50, 06C15

The paper has been supported by the grant VEGA No. 2/0069/16 SAV and GAČR 15-15286S.
} 
of observables was introduced, Dvu6, which was inspired by an order of operators presented by Olson, see [Ols].

There are two very important classes of effect algebras: The first class consists of effect algebras with the Riesz decomposition property (RDP for short), i.e. a joint refinement of any two decompositions is possible. The second class is formed by homogeneous effect algebras, introduced in Jen. It contains the first class as well as orthoalgebras and lattice ordered effect algebras. These effect algebras will be central in our reasoning.

The aim of the present paper is to extend our knowledge on observables. In particular, we show a one-to-one relation between observables and their spectral resolutions, or we describe orthoalgebraic skeleton of homogeneous monotone $\sigma$ complete effect algebras.

The paper is organized as follows. Section 2 gathers the basic notions of the theory of effect algebras. Section 3 defines spectral resolutions. We show when a spectral resolution completely determines an observable. In Section 4, we describe the set of sharp elements of a monotone $\sigma$-complete homogeneous effect algebra; sharp elements will form the orthoalgebraic skeleton of an effect algebra. We note that $\mathcal{P}(H)$ is the orthoalgebraic skeleton of $\mathcal{E}(H)$. In addition, we show when effect algebras satisfy the Observable Existence Property.

\section{Elements of Effect Algebras}

According to $\mathrm{FoBe}$, we say that an effect algebra is a partial algebra $E=$ $(E ;+, 0,1)$ with a partially defined operation + and with two constant elements 0 and 1 such that, for all $a, b, c \in E$,

(i) $a+b$ is defined in $E$ if and only if $b+a$ is defined, and in such a case $a+b=b+a$

(ii) $a+b$ and $(a+b)+c$ are defined if and only if $b+c$ and $a+(b+c)$ are defined, and in such a case $(a+b)+c=a+(b+c)$;

(iii) for any $a \in E$, there exists a unique element $a^{\prime} \in E$ such that $a+a^{\prime}=1$;

(iv) if $a+1$ is defined in $E$, then $a=0$.

If we define $a \leq b$ if and only if there exists an element $c \in E$ such that $a+c=b$, then $\leq$ is a partial ordering on $E$, and we write $c:=b-a$. It is clear that $a^{\prime}=1-a$ for all $a \in E$, and if $a \leq b$, then $b-a=\left(b^{\prime}+a\right)^{\prime}$. For more information about effect algebras, see $\mathrm{DvPu}$. A homomorphism from an effect algebra $E_{1}$ into another one $E_{2}$ is any mapping $h: E_{1} \rightarrow E_{2}$ such that (i) $h(1)=1$ (ii) if $a+b$ is defined in $E_{1}$ so is defined $h(a)+h(b)$ in $E_{2}$ and $h(a+b)=h(a)+h(b)$. A subset $F$ of an effect algebra $E$ is a sub-effect algebra of $E$ if (i) $0,1 \in F$, (ii) if $a \in F$, then $a^{\prime} \in F$, and (iii) if $a, b \in F$ and $a+b \in E$, then $a+b \in F$.

If $G$ is an Abelian partially ordered group written additively, choose an element $u \in G^{+}:=\{g \in G: g \geq 0\}$, and set $\Gamma(G, u):=[0, u]=\{g \in G: 0 \leq g \leq u\}$. Then $(\Gamma(G, u) ;+, 0, u)$ is an effect algebra, where + is the group addition of elements from $\Gamma(G, u)$ if it exists in $\Gamma(G, u)$. Effect algebras that are isomorphic to some $\Gamma(G, u)$ are said to be interval effect algebras. For more information on Abelian partially ordered groups see [Go]. A sufficient condition to be an effect algebra an interval one is the Riesz Decomposition Property, $\operatorname{Rav}$. We say that an effect algebra $E$ satisfies the Riesz Decomposition Property (RDP for short), if $a_{1}+a_{2}=b_{1}+b_{2}$ implies that there are four elements $c_{11}, c_{12}, c_{21}, c_{22} \in E$ such that $a_{1}=c_{11}+c_{12}$, $a_{2}=c_{21}+c_{22}, b_{1}=c_{11}+c_{21}$ and $b_{2}=c_{12}+c_{22}$. Equivalently, $E$ has RDP iff 
$a \leq b+c$ implies that there are $b_{1}, c_{1} \in E$ such that $b_{1} \leq b, c_{1} \leq c$ and $a=b_{1}+c_{1}$. An effect algebra $E$ has the Riesz Interpolation Property (RIP for short) if, given $x_{1}, x_{2}, y_{1}, y_{2}$ in $E$ such that $x_{i} \leq y_{j}$ for all $i, j=1,2$, there exists an element $z \in E$ such that $x_{i} \leq z \leq y_{j}$ for all $i, j=1,2$. We note that RDP implies RIP, but the converse is not always true.

An important class of effect algebras consists of clans which are families of functions where all algebraic operations are defined by points. A clan is a family $\mathcal{C}$ of fuzzy sets $(=[0,1]$-valued functions) on a set $\Omega \neq \emptyset$ such that (i) $1 \in \mathcal{C}$, (ii) if $f \in \mathcal{C}$, then $1-f \in \mathcal{C}$, (iii) if $f, g \in \mathcal{C}, f \leq 1-g$, then $f+g \in \mathcal{C}$.

An effect algebra $E$ is monotone $\sigma$-complete if, for any sequence $a_{1} \leq a_{2} \leq \cdots$, the element $a=\bigvee_{n} a_{n}$ is defined in $E$ (we write $\left\{a_{n}\right\} \nearrow a$ ). If an effect algebra is a lattice or a $\sigma$-lattice or a complete lattice, we say that $E$ is a lattice effect algebra, a $\sigma$-lattice effect algebra, and a complete lattice effect algebra, respectively.

If $E$ and $F$ are two monotone $\sigma$-complete effect algebras, a homomorphism $h$ : $E \rightarrow F$ is said to be a $\sigma$-homomorphism if $\left\{a_{n}\right\} \nearrow a$ implies $\left\{h\left(a_{n}\right)\right\} \nearrow h(a)$ for $a, a_{1}, \ldots \in E$.

Interesting examples of monotone $\sigma$-complete effect algebras are effect-tribes. We remind that an effect-tribe is any system $\mathcal{T}$ of $[0,1]$-valued functions on $\Omega \neq \emptyset$ such that (i) $1 \in \mathcal{T}$, (ii) if $f \in \mathcal{T}$, then $1-f \in \mathcal{T}$, (iii) if $f, g \in \mathcal{T}, f \leq 1-g$, then $f+g \in \mathcal{T}$, and (iv) for any sequence $\left\{f_{n}\right\}$ of elements of $\mathcal{T}$ such that $f_{n} \nearrow f$ (pointwise), then $f \in \mathcal{T}$. It is evident that any effect-tribe is a monotone $\sigma$-complete effect algebra where all algebraic operations are defined by points. We note that $\mathcal{E}(H)$ is isomorphic to the effect-tribe $\mathcal{T}(H)=\{(A \phi, \phi): \phi \in H,\|\phi\|=1, A \in \mathcal{E}(H)\}$ and analogously, the same is true for $\mathcal{P}(H)$.

An effect algebra $E$ is an orthoalgebra if the existence of $a+a$ implies $a=0$. An orthoalgebra $E$ is an orthomodular poset (OMP for short) if the existence of $a+b$ implies $a \vee b$ exists and in such a case $a+b=a \vee b$, [DvPu, Prop 1.5.6]. An orthoalgebra $E$ is an orthomodular poset iff the existence of $a+b, a+c, b+c$ implies $a+b+c$ is defined in $E$, [ $\mathrm{DvPu}$, Thm 1.5.5] or [FGR, Thm 2.12]. If an OMP is also a lattice, we call it an orthomodular lattice (OML for short).

A more general notion than an effect algebra with RDP is the following notion Jen: We say that an effect algebra $E$ is homogeneous if, whenever $a, b, c \in E$ are such that $a \leq b+c, a \leq(b+c)^{\prime}$, there are $a_{1}, a_{2} \in E$ such that $a_{1} \leq b, a_{2} \leq c$ and $a=a_{1}+a_{2}$. We notice that (1) every effect algebra with RDP is homogeneous, (2) every lattice effect algebra is homogeneous, (3) every orthoalgebra is homogenous, (4) every homogeneous effect algebra can be covered by a system of sub-effect algebras $\left(E_{t}: t \in T\right)$ of $E$ such that every $E_{t}$ satisfies RDP, Jen, Thm 3.1, Cor 3.13].

A finite sequence $\left(a_{1}, \ldots, a_{n}\right)$ of elements of $E$ is summable if $a:=a_{1}+\cdots+a_{n}:=$ $\sum_{n=1}^{n} a_{i}$ exists, and the element $a$ is said to be the sum of $\left(a_{1}, \ldots, a_{n}\right)$. An arbitrary family $\left(a_{t}: t \in T\right)$ of elements of $E$ is said to be summable if every finite subsystem of $\left(a_{t}: t \in T\right)$ is summable. If, in addition, there exists $a:=\bigvee\left\{\sum\left\{a_{t}: t \in S\right\}: S\right.$ is a finite subset of $T\}$ in $E$, the element $a$ is said to be the sum of $\left(a_{t}: t \in T\right)$, and we write $a=\sum_{t \in T} a_{t}$.

Two elements $a$ and $b$ of an effect algebra $E$ are said to be (i) compatible and we write $a \leftrightarrow b$ if there exist three elements $a_{1}, b_{1}, c \in E$ such that $a=a_{1}+c$, $b=b_{1}+c$ and $a_{1}+b_{1}+c \in E$, (ii) strongly compatible and we write $a \stackrel{\mathrm{c}}{\longleftrightarrow} b$ if there are three elements $a_{1}, b_{1}, c \in E$ such that $a=a_{1}+c, b=b_{1}+c, a_{1} \wedge b_{1}=0$ and 
$a_{1}+b_{1}+c \in E$. We note that $a \stackrel{\mathrm{c}}{\longleftrightarrow} b$ implies $a \leftrightarrow b$, but the converse implication does not hold, in general.

We say that an arbitrary subset $M$ of $E$ is (i) compatible if, for any finite subset $M_{F}$ of $M$, there is a summable sequence $\left(c_{1}, \ldots, c_{k}\right)$ of elements of $E$ such that every element of $M_{F}$ can be expressed as a sum of some elements from $\left\{c_{1}, \ldots, c_{k}\right\}$; (ii) internally compatible if, for any finite subset $M_{F}$ of $M$, there is a summable sequence $\left(c_{1}, \ldots, c_{k}\right)$ of elements of $M$ such that every element of $M_{F}$ can be expressed as a sum of some elements from $\left\{c_{1}, \ldots, c_{k}\right\}$.

A block of an effect algebra $E$ is a maximal sub-effect algebra, $B$, of $E$ (if it exists in $E$ ) satisfying RDP. If $E$ is a homogeneous effect algebra, then a subset $B$ of $E$ is a block iff $B$ is a maximal internally compatible set with $1 \in B$, Jen, Thm 3.11], and $E$ can be covered by its blocks, [Jen, Cor 3.13].

A state on an effect algebra $E$ is any mapping $s: E \rightarrow[0,1]$ such that (i) $s(1)=1$, and (ii) $s(a+b)=s(a)+s(b)$ whenever $a+b$ is defined in $E$. A state $s$ is $\sigma$-additive if (iii) if $a_{n} \nearrow a$, then $s(a)=\lim _{n} s\left(a_{n}\right)$. Equivalently, a state $s$ is $\sigma$-additive if $a=\sum_{n} a_{n}$ for a sequence of summable elements $\left\{a_{n}\right\}$, then $s(a)=\sum_{n} s\left(a_{n}\right)$. For every state $s$ we have (i) $s\left(a^{\prime}\right)=1-s(a)$, (ii) $a \leq b$ implies $s(a) \leq s(b)$.

A non-empty system of states $\mathcal{S}$ is (i) order-determining if $s(a) \leq s(b)$ for each $s \in \mathcal{S}$ implies $a \leq b$, (ii) full if, for any $a>0$, there is $s \in \mathcal{S}$ such that $s(a)=1$. For example, if $E=\mathcal{E}(H)$ and $s_{\phi}(A):=(A \phi, \phi), A \in \mathcal{E}(H)$, where $\phi$ is any unit vector in $H$, then $\mathcal{S}_{v}(H):=\left\{s_{\phi}: \phi \in H,\|\phi\|=1\right\}$ is an order-determining system of $\sigma$-additive states on $\mathcal{E}(H)$ whilst it is not full; take e.g. $A=1 / 2 I$. A state $s_{\phi}$ is said to be a vector state or a pure state. The restriction of $s_{\phi}$ onto $\mathcal{P}(H)$ is also a $\sigma$-additive state and we will denote it also as $s_{\phi}$. The system $\mathcal{S}_{v}(H)$ is for $\mathcal{P}(H)$ order-determining as well as full. Similarly, if $\mathcal{T}$ is an effect-tribe, $s_{\omega}(f):=f(\omega)$, $f \in \mathcal{T}(\omega \in \Omega)$, then $\left\{s_{\omega}: \omega \in \Omega\right\}$ is an order-determining system of $\sigma$-additive states on $\mathcal{T}$ but not necessarily full.

We note that if an effect algebra $E$ has a full system of states, then $x+x \in E$ implies $x=0$. Indeed, $x+x$ means $x \leq x^{\prime}$. If $x>0$, there is a state $s$ such that $s(x)=1$ which entails $s(x) \leq s\left(x^{\prime}\right)=1$, i.e. $s(x)=0$, a contradiction. In other words, an effect algebra with a full system of states is an orthoalgebra, DvPu, Lem 1.5.2].

An element of an effect algebra $E$ is said to be sharp if $a \wedge a^{\prime}$ exists in $E$ and $a \wedge a^{\prime}=0$. Let $\operatorname{Sh}(E)$ be the set of sharp elements of $E$. Then (i) $0,1 \in \operatorname{Sh}(E)$, (ii) if $a \in \operatorname{Sh}(E)$, then $a^{\prime} \in \operatorname{Sh}(E)$. If $E$ is a lattice effect algebra, then $\operatorname{Sh}(E)$ is an orthomodular lattice which is a sub-effect algebra and a sublattice of $E$, JeRi. If an effect algebra $E$ satisfies RDP, then by Dvu4, Thm 3.2], $\operatorname{Sh}(E)$ is even a Boolean algebra. If $E$ is a homogenous effect algebra, then $\operatorname{Sh}(E)$ is a sub-effect algebra of $E$, [Jen, Cor 4.4], and moreover, $\operatorname{Sh}(E)$ is an orthoalgebra.

An element $a \in E$ is said to be principal if $x, y \leq a$ and $x+y \in E$ imply $x+y \leq a$. A principal element $a \in E$ is said to be central, if for each $b \in E$, there are unique elements $b_{1}, b_{2} \in E$ such that $b_{1} \leq a$ and $b_{2} \leq a^{\prime}$ and $b=b_{1}+b_{2}$. We denote by $C(E)$ the set of central elements of $E$. We note that an element $a$ of an effect algebra $E$ is central iff there exists an isomorphism $f_{a}: E \rightarrow[0, a] \times\left[0, a^{\prime}\right]$ such that $f_{a}(a)=(a, 0)$ and if $f_{a}(b)=\left(b_{1}, b_{2}\right)$, then $b=b_{1}+b_{2}$ for any $b \in E$. Then $C(E)$ is a Boolean algebra such that (i) $0,1 \in C(E)$, (ii) $a \in C(E)$ implies $a^{\prime} \in C(E)$, and (iii) for each $a \in C(E)$ and each $x \in E, a \wedge x$ and $a \vee x$ exist in $E$, 
and $(x \vee a)-x=a-(a \wedge x)$, and (iv) if $E$ is monotone $\sigma$-complete, then $C(E)$ is a Boolean $\sigma$-algebra, Dvu4, Thm 5.11].

We note that $C(\mathcal{E}(H))=\{O, I\}=C(\mathcal{P}(H))$ and $\operatorname{Sh}(\mathcal{E}(H))=\mathcal{P}(H)$, Dvu1, Thm 4.4]. In addition, $\mathcal{P}(H)$ is a sub-effect algebra of $\mathcal{E}(H)$ and $\mathcal{P}(H)$ is a complete (orthomodular) lattice such that suprema and infima of projections on $H$ are the same in both effect algebras. We note that $\mathcal{P}(H)$ is the greatest sub-orthoalgebra (sub-orthomodular poset or sub-orthomodular lattice) of $\mathcal{E}(H)$ containing $\mathcal{P}(H)$. Moreover, RDP fails in $\mathcal{E}(H)$ as well as in $\mathcal{P}(H)$, and $\mathcal{E}(H)$ is homogeneous iff $\operatorname{dim} H \leq 1$, ]Jen, Cor 5.5].

\section{Spectral Resolution and Observables}

In the section, we define observables, and we point out to their very close connection with spectral resolutions. We show when a spectral resolution determines uniquely an observable. We introduce the Observable Existence Property for effect algebras; it guarantees the existence of an observable corresponding to a given spectral resolution.

Let $E$ be a monotone $\sigma$-complete effect algebra. An observable on $E$ is any mapping $x: \mathcal{B}(\mathbb{R}) \rightarrow E$, where $\mathcal{B}(\mathbb{R})$ is the Borel $\sigma$-algebra of the real line $\mathbb{R}$, such that (i) $x(\mathbb{R})=1$, (ii) if $A, B \in \mathcal{B}(\mathbb{R}), A \cap B=\emptyset$, then $x(A \cup B)=x(A)+x(B)$, and (iii) if $\left\{A_{i}\right\}$ is a sequence of Borel sets such that $E_{i} \subseteq E_{i+1}$ for each $i$ and $\bigcup_{i} A_{i}=A$, then $x(A)=\bigvee_{i} x\left(A_{i}\right)$. The set $\mathcal{R}(x)=\{x(A): A \in \mathcal{B}(\mathbb{R})\}$ is said to be the range of an observable $x$.

Observe that any observable is a $\sigma$-homomorphism of monotone $\sigma$-complete effect algebras. The basic properties of observables are (i) $x(\mathbb{R} \backslash A)=x(A)^{\prime}$, (ii) $x(\emptyset)=$ 0 , (iii) $x(A) \leq x(B)$ whenever $A \subseteq B$, and $x(B \backslash A)=x(B)-x(A)$, (iv) if $\left\{A_{i}\right\} \searrow A$, then $x(A)=\bigwedge_{i} x\left(A_{i}\right),(\mathrm{v})$ if $x(A)=x(B)=0$, then $x(A \cup B)=$ $x(A \backslash B)+x(B \backslash A)+x(A \cap B)=0$, (vi) if $x\left(A_{n}\right)=0$ for each $n \geq 1$, then $x\left(\bigcup_{n} A_{n}\right)=0$, (vii) $x(A)+x(B)$ exists iff does $x(A \cup B)+x(A \cap B)$, and in such a case, $x(A)+x(B)=x(A \cup B)+x(A \cap B)$, (viii) $x(A \cup B)-x(A)=x(B)-x(A \cap B)$, (ix) if $x(B)=0$, then $x(A \cup B)=x(A)$, (x) if $x(B)=1$, then $x(A)=x(A \cap B)$, and (xi) $x(A) \leftrightarrow x(B)$. (xi) $\mathcal{R}(x)$ is an internally compatible subset of $E$. Indeed, given a Borel set $A$, we define $A^{1}:=A$ and $A^{0}:=\mathbb{R} \backslash A$. If $A_{1}, \ldots, A_{n}$ are Borel sets, for any sequence $\left(i_{1}, \ldots, i_{n}\right)$ of 0 's and 1 's, let $A\left(i_{1}, \ldots, i_{n}\right):=A_{1}^{i_{1}} \cap \cdots \cap A_{n}^{i_{n}}$. Then $\left\{x\left(A\left(i_{1}, \ldots, i_{n}\right)\right):\left(i_{1}, \ldots, i_{n}\right) \in\{0,1\}^{n}\right\}$ is a summable system of elements from $\mathcal{R}(x)$ and each element $x\left(A_{i}\right)$ is a sum of some finitely many elements of the summable system.

The least closed subset $C$ of $\mathcal{B}(\mathbb{R})$ is said to be the spectrum of $x$, and we denote it by $\sigma(x)$; since the natural topology of the real line satisfies the second countability axiom, $\sigma(x)$ exists, and $x(\sigma(x))=1$. In addition, a point $\lambda \in \mathbb{R}$ belongs to $\sigma(x)$ iff for any open set $U$ containing $\lambda, x(U) \neq 0$. An observable $x$ is: (i) bounded, if $\sigma(x)$ is a compact set, (ii) bounded from above (from below), if there is $k \in \mathbb{R}$ such that $\sigma(x) \subseteq(-\infty, k](\sigma(x) \subseteq[k, \infty))$, (iii) positive (negative) if $\sigma(x) \subseteq[0, \infty)$ $(\sigma(x) \subseteq(-\infty, 0])$, (iv) a question if $\sigma(x) \subseteq\{0,1\}$, (v) simple if $\sigma(x)$ is a finite non-empty subset of $\mathbb{R}$, and (vi) an effect observable if $\sigma(x) \subseteq[0,1]$.

Given a bounded observable $x$, we define the norm of $x$ as $\|x\|:=\sup \{|\lambda|: \lambda \in$ $\sigma(x)\}$. Then $x=q_{0}$ iff $\|x\|=0$.

If $x$ is an observable and $f: \mathbb{R} \rightarrow \mathbb{R}$ is a Borel measurable function, then the mapping $f \circ x: A \mapsto x\left(f^{-1}(A)\right), A \in \mathcal{B}(\mathbb{R})$, is also an observable. If, e.g. $f(t)=t^{n}$, 
$t \in \mathbb{R}$, then we write $f \circ x=x^{n}$. It is possible to show that if $x$ is a bounded observable and $f$ is continuous on $\sigma(x)$, then $\sigma(f \circ x)=f(\sigma(x))$.

We denote by $\mathcal{O}(E), \mathcal{B O}(E)$ and $\mathcal{E} \mathcal{O}(E)$ the set of all observables, bounded observables and the set of effect observables, respectively, on $E$.

For example, let $\left\{a_{n}\right\}$ be a finite or infinite sequence of summable elements, $\sum_{n} a_{n}=1$, and let $\left\{t_{n}\right\}$ be a sequence of mutually different real numbers. Then the mapping $x: \mathcal{B}(\mathbb{R}) \rightarrow E$ defined by

$$
x(A):=\sum\left\{a_{n}: t_{n} \in A\right\}, A \in \mathcal{B}(\mathbb{R}),
$$

is an observable on $E$. In particular, if $t_{0}=0, t_{1}=1$ and $a_{0}=a^{\prime} a_{1}=a$ for some fixed element $a \in E, x$ defined by (3.0) is an observable, called a question corresponding to the element $a$, and we write $x=q_{a}$.

The following characterization of question observables was known for observables on $\sigma$-orthomodular lattices, see e.g. Gud, Lem 3.5(iii)]. Here we generalize it as follows:

Lemma 3.1. A bounded observable $x$ on a monotone $\sigma$-complete effect algebra $E$ is a question observable if and only if $x=x^{2}$.

Proof. Clearly, if $\sigma(x) \subseteq\{0,1\}$, then $x=x^{2}$. Conversely, assume that $x=x^{2}$. Then $x$ is positive, so that $x((-\infty, 0))=0$. Choose a rational $t>1$. Then $t<t^{2}$ so that $x\left(\left[t^{2}, \infty\right)\right)=x^{2}\left(\left[t^{2}, \infty\right)\right)=x([t, \infty))$ which yields $x\left(\left[t, t^{2}\right)\right)=x\left(\left[t^{2}, \infty\right)\right)-$ $x([t, \infty))=0$. In a similar way, we have $x\left(\left[t^{2^{n}}, t^{2^{n+1}}\right)\right)=0$ for each $n \geq 1$. Hence, $x([t, \infty))=\sum_{n=0}^{\infty} x\left(\left[t^{2^{n}}, t^{2^{n+1}}\right)=0\right.$, and since $t>1$ was arbitrary, we have $x((0, \infty))=\bigvee_{t \searrow 0} x((t, \infty))=0$.

Now let a rational number $t$ be such that $0<t<1$. Then $t^{2}<t$, so that $x\left(\left[t^{2}, t\right)\right)=0$, and in a similar way, we have $x\left(\left[t^{2^{n+1}}, t^{2^{n}}\right)\right)=0$ for each $n \geq 1$. Therefore, $x((0, t))=0$. Since $t<1$ was arbitrary, we have $x((0,1))=0$.

Finally, we have $\sigma(x) \subseteq\{0,1\}$.

We present a simple but useful proposition, see also [DvKu, Rem 3.3]:

Proposition 3.2. Let $x$ be an observable on a monotone $\sigma$-complete effect algebra $E$. Given a real number $t \in \mathbb{R}$, we put

$$
x_{t}:=x((-\infty, t)) .
$$

Then

$$
\begin{gathered}
x_{t} \leq x_{s} \quad \text { if } t<s, \\
\bigwedge_{t} x_{t}=0, \quad \bigvee_{t} x_{t}=1,
\end{gathered}
$$

and

$$
\bigvee_{t<s} x_{t}=x_{s}, s \in \mathbb{R}
$$

Conversely, if there is a system $\left\{x_{t}: t \in \mathbb{R}\right\}$ of elements of $E$ satisfying (3.2)(3.4) and if there is an observable $x$ on $E$ for which (3.1) holds for any $t \in \mathbb{R}$, then $x$ is unique. 
Proof. Since $E$ is a monotone $\sigma$-complete effect algebra, from (3.2) we conclude that (3.4) holds. Indeed, if $\left\{t_{n}\right\} \nearrow s$ and $\left\{t_{n}^{\prime}\right\} \nearrow s$ are two sequences, then $\bigvee_{n} x_{t_{n}}=x_{s}=\bigvee_{n} x_{t_{n}^{\prime}}=\bigvee_{t<s} x_{t}$. Similarly for the other properties.

Now assume that $x$ is an observable such that $x((-\infty, t))=x_{t}$ for each $t \in \mathbb{R}$. Let $y$ be another observable of $E$ such that $y((-\infty, t))=x_{t}$ for each $t \in \mathbb{R}$. We denote by $\mathcal{K}=\{A \in \mathcal{B}(\mathbb{R}): x(A)=y(A)\}$. Then $\mathcal{K}$ is a Dynkin system, i.e. a system of subsets containing its universe which is closed under the set theoretical complements and countable unions of disjoint subsets, $\mathrm{Bau}$. It contains all intervals of the form $(-\infty, t)$ for each $t \in \mathbb{R}$; these intervals form a $\pi$-system, i.e. intersection of any two sets from the $\pi$-system is from the $\pi$-system. Hence by the Sierpiński Theorem, Kal, Thm 1.1], $\mathcal{K}$ is also a $\sigma$-algebra, and consequently, we have $\mathcal{K}=\mathcal{B}(\mathbb{R})$.

It is worthy to recall the following note. Let $\left\{x_{t}: t \in \mathbb{R}\right\}$ satisfy (3.2)-(3.4), $\mathbb{R}^{*}=\mathbb{R} \cup\{-\infty\} \cup\{\infty\}$, and $x_{-\infty}=0$ and $x_{\infty}=1$. Define $M=\left\{x_{t}-x_{s}: s, t \in\right.$ $\left.\mathbb{R}^{*}, s \leq t\right\}$. Then $M$ is internally compatible. (The same is true if we change $\mathbb{R}$ to $\mathbb{Q}$.) Indeed, let $F=\left\{x_{t_{1}}-x_{s_{i}}, \ldots, x_{t_{n}}-x_{s_{n}}\right\}$, where $s_{i} \leq t_{i}$ and $t_{i}, s_{i} \in \mathbb{R}^{*}$ for $i=1, \ldots, n$. Then $\left\{t_{1}, s_{2}, \ldots, t_{n}, s_{n}\right\}=\left\{u_{1}, \ldots, u_{m}\right\}$, where $u_{1}<\cdots<u_{m}$. Then $S=\left\{x_{u_{1}}, x_{u_{2}}-x_{u_{1}}, \ldots, x_{u_{m}}-x_{u_{m-1}}\right\}$ is a summable system of $E$ and every element $x_{t_{i}}-x_{s_{i}}$ for $i=1, \ldots, n$, is a sum of some finitely many elements of $S$.

The system $\left\{x_{t}: t \in \mathbb{R}\right\}$ from Proposition 3.2 satisfying (3.2)-(3.4) is said to be the spectral resolution of an observable $x$. To represent observables by their spectral resolutions and vice-versa, we have to establish conditions when a given system $\left\{x_{t}: t \in \mathbb{R}\right\}$ of elements of $E$ satisfying (3.2)-(3.4) determines an observable $x$ such that $x((-\infty, t))=x_{t}, t \in \mathbb{R}$. By Proposition 3.2, it is enough to find at least one observable $x$, then it is unique. Some such conditions were established in DvKu, Dvu5.

Motivated by this problem, we say that a monotone $\sigma$-complete effect algebra $E$ has the Observable Existence Property (OEP, for short) if given a system of elements $\left\{x_{t}: t \in \mathbb{R}\right\}$ of $E$ satisfying (3.2)-(3.4), there is an observable $x$ such that $x((-\infty, t))=x_{t}, t \in \mathbb{R}$. We denote by $\mathcal{O E P}(E A)$ the class of effect algebras with OEP. The class $\mathcal{O E P}(E A)$ contains these effect algebras:

(i) $\sigma$-MV-algebras, $\mathrm{DvKu}, \mathrm{Thm} 3.2]$.

(ii) $\sigma$-lattice effect algebras, $\mathrm{DvKu}, \mathrm{Thm} 3.5]$.

(iii) Boolean $\sigma$-algebras, [DvKu, Thm 3.6].

(iv) $\sigma$-orthocomplete orthomodular posets, [DvKu, Thm 3.8].

(v) Monotone $\sigma$-complete effect algebras with RDP, [DvKu, Thm 3.9].

(vi) $\mathcal{E}(H)$, DvKu, Thm 3.10].

(vii) Effect-tribes, DvKu, Thm 3.11].

(viii) Monotone $\sigma$-complete effect algebras with RIP and DMP, [Dvu5, Thm 4.3].

We do not know whether every monotone $\sigma$-complete effect algebra belongs to the class $\mathcal{O E P}(E A)$.

A partial answer to this problem is the following result. First we define a kind of representable monotone $\sigma$-complete effect algebras. Another type of representable effect algebras was defined in Dvu5.

We say that a monotone $\sigma$-complete effect algebra $E$ is representable if there is an effect-tribe of fuzzy sets $\mathcal{T}$ and a $\sigma$-homomorphism $h$ of monotone $\sigma$-complete effect algebras from $\mathcal{T}$ onto $E$ such that if $f, g \in \mathcal{T}$ with $f \leq g$ and if there is $c_{1} \in E$ such that $h(f) \leq c_{1} \leq h(g)$, then there is $c \in \mathcal{T}$ such that $h(c)=c_{1}$ and $f \leq c \leq g$. 
For example, every (1) monotone $\sigma$-complete effect algebra with RDP ([BCD] ) and [DvKu, Thm 3.9, Claim 2], (2) $E$ which is a $\sigma$-MV-algebra Dvu2, (3) $\mathcal{E}(H)$, , (4) effect-tribe is representable. Representable monotone $\sigma$-complete effect algebras are connected with the Loomis-Sikorski representation of monotone $\sigma$-complete effect algebras by effect-tribes, see e.g. BCD, Dvu2, Mun.

We note that every monotone $\sigma$-complete effect algebra $E$ with an order-determining system $\mathcal{S}$ of $\sigma$-additive states is representable. Indeed, for any $a \in E$, we define a function $\bar{a}: \mathcal{S} \rightarrow[0,1]$ such that $\bar{a}(s):=s(a), s \in \mathcal{S}$. Then $\bar{E}:=\{\bar{a}: a \in E\}$ is an effect-tribe that is $\sigma$-isomorphic to $E$.

In addition, every monotone $\sigma$-complete effect algebra $E$ is representable if $E$ possesses an order-determining system of states: The family $\bar{E}:=\{\bar{a}: a \in E\}$ is a clan isomorphic to $E$ that is even an effect-tribe. Indeed, let $\left\{\bar{a}_{n}\right\}$ be a nondecreasing sequence of elements of $\bar{E}$. Then $\left\{a_{n}\right\}$ is a non-decreasing sequence of elements of $E$, so that there is $a \in E$ such that $\left\{a_{n}\right\} \nearrow a$. Then $\bar{a}_{n} \leq \bar{a}$. If there is $b \in E$ such that $\bar{a}_{n} \leq \bar{b}$ for each $n \geq 1$, then $a_{n} \leq b$ and $a \leq b$ which yields $\bar{a} \leq \bar{b}$. Consequently, $E$ is $\sigma$-isomorphic to the effect-tribe $\bar{E}$.

In the next result we show that every representable monotone $\sigma$-complete effect algebra belongs to the class $\mathcal{O E P}(E A)$.

Theorem 3.3. Every representable monotone $\sigma$-complete effect algebra satisfies OEP.

Proof. Let $\left\{x_{t}: t \in \mathbb{R}\right\}$ be a system of elements of $E$ satisfying (3.2)-(3.4). Let $r_{1}, r_{2}, \ldots$ be any enumeration of the set of rational numbers. Due to the assumptions, there are a tribe $\mathcal{T}$ of fuzzy functions from $[0,1]^{\Omega}$ for some non-void set $\Omega$ and a $\sigma$-homomorphism $h$ from $\mathcal{T}$ onto $E$ such that if $f, g \in \mathcal{T}$ with $f \leq g$ are given and if there is $c_{1} \in E$ with $h(f) \leq c_{1} \leq h(g)$, there is $c \in \mathcal{T}$ such that $f \leq c \leq g$ and $h(c)=c_{1}$. Given $r_{n}$, let $a_{n}$ be a function from the tribe $\mathcal{T}$ such that $h\left(a_{n}\right)=x_{r_{n}}$ for any $n \geq 1$. We are claiming that it is possible to find such a sequence of functions $\left\{b_{n}\right\}$ from $\mathcal{T}$ such that $h\left(b_{n}\right)=x_{r_{n}}$ for any $n \geq 1$ and $b_{n} \leq b_{m}$ whenever $r_{n}<r_{m}$. Indeed, if $n=1$, we set $b_{1}=a_{1}$. By mathematical induction suppose that we have find $b_{1}, \ldots, b_{n}$ such that $h\left(b_{i}\right)=x_{r_{i}}$ and $b_{i} \leq b_{j}$ whenever $r_{i}<r_{j}$ for $i, j=1, \ldots, n$. Let $j_{1}, \ldots, j_{n}$ be a permutation of $1, \ldots, n$ such that $r_{j_{1}}<\cdots<r_{j_{n}}$. For $r_{n+1}$ we have three possibilities (i) $r_{n+1}<r_{j_{1}}$, (ii) there exists $k=1, \ldots, n-1$ such that $r_{j_{k}}<r_{n+1}<r_{j_{k+1}}$, or (iii) $r_{j_{n}}<r_{n+1}$. Applying the assumption, we can find $b_{n+1} \in \mathcal{T}, h\left(b_{n+1}\right)=r_{n+1}$, such that for all $i, j=1, \ldots, n+1, b_{i} \leq b_{j}$ whenever $r_{i}<r_{j}$.

Thus, we can assume that the sequence of functions $\left\{b_{r_{n}}\right\}$, where $b_{r_{n}}:=b_{n}$ for $n \geq 1$, is linearly ordered. Using the density of rational numbers in $\mathbb{R}$, for any real number $t \in \mathbb{R}$, we can find a function $b_{t} \in \mathcal{T}$ such that $h\left(b_{t}\right)=x_{t}$. Indeed, if $\left\{p_{n}\right\} \nearrow t$ and $\left\{q_{n}\right\} \nearrow t$ for two sequences of rational numbers, $\left\{p_{n}\right\}$ and $\left\{q_{n}\right\}$, we can show that $\bigvee_{n} b_{p_{n}}=\bigvee_{n} b_{q_{n}}$. Hence, $b_{t}:=\bigvee_{n} b_{r_{n}}$ is a well-defined element of $\mathcal{T}$ satisfying $h\left(b_{t}\right)=r_{t}$. In addition, the system of functions $\left\{b_{t}: t \in \mathbb{R}\right\}$ is also linearly ordered, and $b_{t} \leq b_{s}$ if $t<s$.

Let $\omega$ be a fixed element of the set $\Omega$. We define $F_{\omega}(t):=b_{t}(\omega), t \in \mathbb{R}$. It is clear that $F_{\omega}$ is a nondecreasing, left continuous function such that $\lim _{t \rightarrow-\infty} F_{\omega}(t)=0$ and $\lim _{t \rightarrow \infty} F_{\omega}(t)=1$. By [Hal, Thm 43.2], $F_{\omega}$ is a distribution function on the measurable space $(\mathbb{R}, \mathcal{B}(\mathbb{R}))$ corresponding to a unique probability measure $P_{\omega}$ on $\mathcal{B}(\mathbb{R})$, that is $P_{\omega}((-\infty, t))=F_{\omega}(t)$ for every $t \in \mathbb{R}$. Define now a mapping $\xi: \mathcal{B}(\mathbb{R}) \rightarrow[0,1]^{\Omega}$ by $\xi(A)(\omega)=P_{\omega}(A), A \in \mathcal{B}(\mathbb{R}), \omega \in \Omega$. In particular, we 
have $\xi((-\infty, t))=b_{t} \in \mathcal{T}$ for any $t \in \mathbb{R}$. To prove that every $\xi(A) \in \mathcal{T}$ for any $A \in \mathcal{B}(\mathbb{R})$, let $\mathcal{K}=\{A \in \mathcal{B}(\mathbb{R}): \xi(A) \in \mathcal{T}\}$. Then $\mathcal{K}$ is a Dynkin system containing all intervals of the form $(-\infty, t)$ for $t \in \mathbb{R}$ which form a $\pi$-system. Hence by Kal, Thm 1.1], $\mathcal{K}$ is a $\sigma$-algebra of subsets, so that, $\mathcal{K}=\mathcal{B}(\mathbb{R})$.

Therefore, $\xi$ is an observable on $\mathcal{T}$ and $x:=h \circ \xi$ is an observable on $M$ such that $x((-\infty, t))=x_{t}$ for any $t \in \mathbb{R}$ which proves that $E$ satisfies OEP.

Example 3.4. Let $\Omega$ be a non-empty set and $\mathcal{D}$ be a Dynkin system on $\Omega$. If $f: \Omega \rightarrow \mathbb{R}$ is a $\mathcal{D}$-measurable function, then the mapping $x_{f}: \mathcal{B}(\mathbb{R}) \rightarrow \mathcal{D}$ given by $x_{f}(A):=f^{-1}(A), A \in \mathcal{B}(\mathbb{R})$, is an observable on $\mathcal{D}$, in addition, $\mathcal{S}_{f}:=\left\{f^{-1}(A)\right.$ : $A \in \mathcal{B}(\mathbb{R})\}$ is a $\sigma$-algebra of subsets of $\Omega$. If $s$ is a $\sigma$-additive state on $\mathcal{D}$, then $s_{x_{f}}(A):=s\left(f^{-1}(A)\right), A \in \mathcal{B}(\mathbb{R})$, is a $\sigma$-additive measure on $\mathcal{B}(\mathbb{R})$.

In addition, $\mathcal{D} \in \mathcal{O} \mathcal{E P}(E A)$.

Proof. Let $\left\{x_{t}: t \in \mathbb{R}\right\}$ be a system of subsets from $\mathcal{D}$ satisfying (3.2)-(3.4). Let $r_{1}, r_{2}, \ldots$ be any enumeration of the set real numbers of $\mathbb{R}$. Define a mapping $f: \Omega \rightarrow \mathbb{R}$ by $f(\omega):=\inf \left\{r_{j}: \omega \in x_{r_{j}}\right\}$. Then $f^{-1}\left(\left(-\infty, r_{k}\right)\right)=\bigcup_{i: r_{i}<r_{k}} x_{r_{i}}=$ $x_{r_{k}} \in \mathcal{D}$. So that, the set $\mathcal{K}=\left\{A \in \mathcal{B}(\mathbb{R}): f^{-1}(A) \in \mathcal{D}\right\}$ is a Dynkin system containing all intervals $\left(-\infty, r_{k}\right)$ which is a $\pi$-system. Hence, $\mathcal{K}=\mathcal{B}(\mathbb{R})$, which proves $\mathcal{D} \in \mathcal{O} \mathcal{E P}(E A)$.

Let $x$ be a simple observable with $\sigma(x)=\left\{t_{1}, \ldots, t_{n}\right\}$ for some real numbers $t_{1}<\cdots<t_{n}$. Put $a_{i}=x\left(\left\{t_{i}\right\}\right), i=1, \ldots, n$, then $a_{1}+\cdots+a_{n}=1$, and for the spectral resolution of $x$, we have

$$
x((-\infty, t))= \begin{cases}0 & \text { if } t \leq t_{1}, \\ a_{1}+\cdots+a_{i} & \text { if } t_{i}<t \leq t_{i+1}, i=1, \ldots, n-1, \\ 1 & \text { if } t_{n}<t\end{cases}
$$

for $t \in \mathbb{R}$. In particular, if $q_{a}$ is a question observable corresponding to an element $a \in E$, then the spectral resolution of $q_{a}$ is as follows

$$
q_{a}((-\infty, t))= \begin{cases}0 & \text { if } t \leq 0 \\ a^{\prime} & \text { if } 0<t \leq 1 \\ 1 & \text { if } 1<t\end{cases}
$$

for $t \in \mathbb{R}$.

\section{Sharp Elements and Observables on Orthoalgebras}

We show that the set of sharp elements of a homogeneous monotone $\sigma$-complete effect algebra is a monotone $\sigma$-complete sub-effect algebra. It will describe the so-called orthoalgebraic skeleton. It allows to characterize sharp observables by a sharp spectral resolution. In addition, we show that every monotone $\sigma$-complete orthoalgebra with RIP satisfies the Observable Existence Property.

In [Jen, Cor 4.4], there was proved that if $E$ is a homogeneous effect algebra, then $\operatorname{Sh}(E)$ is a sub-effect algebra of $E$. Now we extend this result for monotone $\sigma$-complete homogeneous effect algebras.

Theorem 4.1. If $E$ is a monotone $\sigma$-complete homogeneous effect algebra, then $\mathrm{Sh}(E)$ is a monotone $\sigma$-complete homogenous sub-effect algebra of $E$. 
Proof. Let $\left\{b_{n}\right\}$ be a sequence of sharp elements of $E$ such that $\left\{b_{n}\right\} \nearrow b$, where $b \in E$. We have to show that $b$ is a sharp element of $E$. We define elements $a_{1}=b_{1}$, and $a_{n}=b_{n}-b_{n-1}$ for $n \geq 2$. Then the sequence $\left\{a_{n}\right\}$ is summable with sum $b=\sum_{n} a_{n}$. The set $M=\left\{b^{\prime}, a_{1}, a_{2}, \ldots\right\}$ is an internally compatible subset of $E$, so there is a block $B$ of $E$ containing $M$. Since $B$ is a sub-effect algebra of $E$ with RDP, we get $b \in B$ and every $b_{n} \in B$. Hence, $b=\bigvee_{B} b_{n}=\bigvee_{n} b_{n}$, where $\bigvee_{B}$ is the join taken in $B$. Since each $b_{n}$ is a sharp element of $E$, so is sharp in the block $B$ which satisfies RDP. This implies that every $b_{n}$ is a central element of the effect algebra $B$. Therefore, $b_{n} \wedge_{B} x$ exists in $B$ for each $x \in B$; here $\wedge_{B}$ means the meet taken in the block $B$, in contrast to $\wedge, \vee$ which denote meet and join taken in the whole $E$.

Given a subset $M$ of $E$, we define inductively a sequence $\left\{M_{n}: n \geq 0\right\}$ of subsets of $E$ as follows: $M_{0}:=M$, and for each $n \geq 1$, we put $M_{n}=\{x \in E: x \leq$ $y, y^{\prime}$ for some $\left.y \in M_{n}\right\} \cup\left\{y-x: x \leq y, y^{\prime}\right.$ for some $\left.y \in M_{n}\right\}$, and for them we put $\bar{M}=\bigcup_{n=0}^{\infty} M_{n}$. If $M$ is a block of $E$, by [Jen, Cor 3.8(b)], $M=\bar{M}$.

Let $x \leq b, b^{\prime}$. Since $B=\bar{B}, x \in B$. First we show that $b_{n} \wedge x=0=b_{n} \wedge_{B} x$ for each $n$. Let $z \leq x, b_{n}$, then $z \leq x \leq b^{\prime} \leq b_{n}^{\prime}$ so that $x=0$. We assert, that there is $x_{0} \in B$ such that $b_{n} \wedge_{B} x \leq x_{0} \leq x, b$ for each $n$. At any rate, the element $x_{0}=0 \in B$ is such an element. Now let $x_{0}$ be any element of $B$ such that $0=b_{n} \wedge_{B} x \leq x_{0} \leq x, b$.

Now we prove a series of Claims.

Claim 1. $x=\left(x-x_{0}\right)+x_{0}, b=\left(b-x_{0}\right)+x_{0},\left(x-x_{0}\right)+\left(b-x_{0}\right)+x_{0} \in E$.

Indeed, we have $x-\left(b_{n} \wedge_{B} x\right)=\left(b_{n} \vee_{B} x\right)-b_{n}$. Since $1=\left(b_{n} \vee_{B} x\right)^{\prime}+\left(\left(b_{n} \vee_{B}\right.\right.$ $\left.x)-b_{n}\right)+b_{n}$, we have $b_{n} \leq\left(x-\left(b_{n} \wedge_{B} x\right)\right)^{\prime} \leq\left(x-x_{0}\right)^{\prime}$ and $b \leq\left(x-x_{0}\right)^{\prime}$ which gives $\left(x-x_{0}\right)+b \in E$. Clearly $x=\left(x-x_{0}\right)+x_{0}, b=\left(b-x_{0}\right)+x_{0}$. In addition, $x, b \leq\left(x-x_{0}\right)+b$.

Claim 2. $x_{0}=0$.

To prove the claim, it is sufficient to show that $\bigwedge_{B, n}\left(x-\left(b_{n} \wedge_{B} x\right)\right)=x-x_{0}=$ $\bigwedge_{n}\left(x-\left(b_{n} \wedge_{B} x\right)\right)$. It is clear that $x-x_{0} \leq x-\left(x \wedge_{B} b_{n}\right)$ for each $n \geq 1$. Let $d \in E$ be such that $d \leq x-\left(x \wedge_{B} b_{n}\right)$ for each $n$. Therefore, $d \leq b, b^{\prime}$ so that $b \in B$. Then $d \leq x-\left(x \wedge_{B} b_{n}\right)=\left(x \vee_{B} b_{n}\right)-b_{n}$ and $d+b_{n} \leq x \vee_{B} b_{n} \leq\left(x-x_{0}\right)+b$ when we have used the end of the proof of Claim 1. Whence, $b_{n} \leq\left(\left(x-x_{0}\right)+b\right)-d$ and $b \leq\left(\left(x-x_{0}\right)+b\right)-d$ which gives $b+d \leq\left(x-x_{0}\right)+b$ and $d \leq x-x_{0}$. Finally $x-x_{0}=\bigwedge_{B, n}\left(x-\left(x \wedge_{B} b_{n}\right)\right)=\bigwedge_{n}\left(x-\left(b_{n} \wedge_{B} x\right)\right)=x$ which gives $x_{0}=0$.

Claim 3. $x \wedge b=0=x \wedge_{B} b$.

Let for $z \in E$, we have $z \leq x, b$. Since $z \leq x \leq b^{\prime}$, we have $z \leq b, b^{\prime}$ and $b \in B$. Then $0=b_{n} \wedge_{B} x \leq z \leq x, b$ which by Claim 2 yields $z=0$.

Finally, since we have $x \leq b, b^{\prime}$, then by Claim 3, we have $x=x \wedge b=0$ and this proves that $b$ is a sharp element of $E$, and consequently, $\operatorname{Sh}(E)$ is a monotone $\sigma$-complete sub-effect algebra which is homogenous because $\operatorname{Sh}(E)$ is an suborthoalgebra of $E$ and every orthoalgebra is homogenous.

Remark 4.2. We note that if $E$ is a homogeneous effect algebra, then $\operatorname{Sh}(E)$ is the biggest orthoalgebra that is a sub-effect algebra of $E$ and every sharp element in the sub-effect algebra is a sharp element of $E$. Indeed, if $E_{0}$ is any orthoalgebra that is a sub-effect algebra of $E$ such that $\operatorname{Sh}\left(E_{0}\right) \subseteq \operatorname{Sh}(E)$, then for each $x \in E_{0}$, we have 
$x \wedge x^{\prime}$ exists in $E$ and is zero, so that $E_{0} \subseteq \operatorname{Sh}(E)$. We call the biggest orthoalgebra of $E$ the orthoalgebraic skeleton of $E$ if it exists in $E$. Under the conditions of Theorem 4.1, $\operatorname{Sh}(E)$ is a monotone $\sigma$-complete orthoalgebraic skeleton of $E$.

We note, as it was already said, that $\mathcal{P}(H)$ is the orthoalgebraic skeleton of $\mathcal{E}(H)$.

Now we characterize sharp observables as follows.

Proposition 4.3. Let $x$ be an observable on a homogenous monotone $\sigma$-complete effect algebra $E$. The following statements are equivalent:

(i) $x$ is a sharp observable.

(ii) $x((-\infty, t)) \in \operatorname{Sh}(E)$ for each $t \in \mathbb{R}$.

(iii) $x((-\infty, t)) \in \operatorname{Sh}(E)$ for each $t \in \mathbb{Q}$.

Proof. Of course (i) implies (ii), and (ii) does (iii).

(ii) $\Rightarrow$ (i). Let $\mathcal{K}:=\{A \in \mathcal{B}(\mathbb{R}): x(A) \in \operatorname{Sh}(E)\}$. We assert that $\mathcal{K}$ is a Dynkin system. Assume $A, B \in \mathcal{K}, A \cap B=\emptyset$. Let $a \in E$ be such that $a \leq x(A \cup B), x((A \cup$ $B)^{c}$. Due to homogeneity of $E$, there are $a_{1}, a_{2} \in E$ with $a_{1} \leq x(A)$ and $a_{2} \leq x(B)$ such that $a=a_{1}+a_{2}$. Then $a_{1} \leq a_{1}+a_{2} \leq(x(A)+x(B))^{\prime} \leq x(A)^{\prime}$ which yields $a_{1}=0$, and in a similar way, we have $a_{2}=0$. Hence, $a=0, x(A \cup B) \in \operatorname{Sh}(E)$, and $A \cup B \in \mathcal{K}$.

Now let $\left\{A_{i}\right\}$ be a sequence of mutually disjoint Borel subsets from $\mathcal{K}$. By the first part of the present proof, $B_{n}=A_{1} \cup \cdots \cup A_{n} \in \mathcal{K}$. Applying Theorem 4.1, we see that $A=\bigcup_{n} A_{n}=\bigcup_{n} B_{n} \in \mathcal{K}$.

Hence, $\mathcal{K}$ is clearly a Dynkin system containing all intervals of the form $(-\infty, t)$, $t \in \mathbb{R}$. These intervals form a $\pi$-system, hence, by the Sierpiński Theorem, Kal, Thm 1.1], $\mathcal{K}$ is a $\sigma$-algebra, which proves $\mathcal{K}=\mathcal{B}(\mathbb{R})$.

(iii) $\Rightarrow$ (ii). Let $t \in \mathbb{R}$. There is a sequence $\left\{r_{n}\right\}$ of rational numbers such that $\left\{r_{n}\right\} \nearrow t$. Applying Theorem 4.1, we see that $x((-\infty, t)) \in \operatorname{Sh}(E)$.

This allows us to show that any spectral resolution consisting of sharp elements of a monotone $\sigma$-complete homogeneous effect algebra always entails the corresponding sharp observable.

If $E_{1}$ is a sub-orthoalgebra of an orthoalgebra $E$ such that $E_{1}$ is a Boolean algebra in its own right, we call $E_{1}$ a Boolean sub-orthoalgebra of $E$. If in addition, $E_{1}$ is a Boolean $\sigma$-algebra in its own right, we call $E_{1}$ a Boolean $\sigma$-sub-orthoalgebra of $E$.

Theorem 4.4. Let $x$ be an observable of a monotone $\sigma$-complete orthoalgebra $E$. Then the range $\mathcal{R}(x)$ is a Boolean $\sigma$-sub-orthoalgebra of $E$.

Proof. (i) The range $\mathcal{R}(x)$ contains 0,1 and is closed under the operation '. Now let $x(G)+x(F)$ exist in $E$. Then $x(G)+x(F)=x(G \backslash F)+x(G \cap F)+x(F \backslash G)+x(G \cap F)$ which means $x(G \cap F)=0$. Therefore, $x(G)+x(F)=x((G \backslash F) \cup(F \backslash G))=$ $x(G \cup F) \in \mathcal{R}(x)$ which shows that the range $\mathcal{R}(x)$ is a sub-orthoalgebra of $E$.

In particular, if $x(A) \leq x(B)$, then $x(B)-x(A)=\left(x(B)^{\prime}+x(A)\right)^{\prime}=x\left(\left(B^{\prime} \cup\right.\right.$ $\left.A)^{\prime}\right)=x(B \backslash A)$. Hence, $x(A \cup B)=x\left((A \cup B) \cap A^{\prime}\right)+x((A \cup B) \cap A)=x(B \backslash$ $A)+x(A)=x(B)$. In a similar way, we can show $x(A)=x(A \cap B)$.

(ii) Now we show that $\mathcal{R}(x)$ is an orthomodular poset in its own right. To show that we have to verify that if $x(A)+x(B), x(A)+x(C), x(B)+x(C)$ exist in $\mathcal{R}(x)$ then $x(A)+x(B)+x(C)$ is defined in $\mathcal{R}(x)$. From the first part of the proof we have $x(A \cap B)=x(A \cap C)=x(B \cap C)=0$. Hence, $x(A)=x(A \backslash(B \cup C))$, 
$x(B)=x(B \backslash(A \cup C))$ and $x(C)=x(C \backslash(A \cup B))$ which yields $x(A \cup B \cup C)=$ $x(A)+x(B)+x(C) \in \mathcal{R}(x)$. Then $a+b=a \vee_{\mathcal{R}(x)} b$ for $a, b \in \mathcal{R}(x)$, where $\vee_{\mathcal{R}(x)}$ is the join taken in $\mathcal{R}(x)$.

(iii) We establish that $\mathcal{R}(x)$ is monotone $\sigma$-complete. To show that, let $\left\{x\left(G_{i}\right)\right\}$ be a sequence of summable elements. According to (ii), we can show that $x\left(G_{1}\right)+$ $\cdots+x\left(G_{n}\right)=x\left(G_{1} \cup \cdots \cup G_{n}\right)$. Hence, if $G=\bigcup_{n} G_{n}$, then $x(G)=\bigvee_{n} x\left(G_{1} \cup \cdots \cup\right.$ $\left.G_{n}\right)=\sum_{n} x\left(G_{n}\right)$.

(iv) In this part we establish that $\mathcal{R}(x)$ satisfies RDP. Let $x(A) \leq x(F)+x(G)$. Then $x(A) \leq x(A)+x(F)=x((F \backslash G) \cup(G \backslash F))$. Hence by the end of (i), $x(A)=x(((F \backslash G) \cup(G \backslash F)) \cap A)=x((F \backslash G) \cap A) \cup((G \backslash F) \cap A))=x((F \backslash G) \cap$ $A)+x((G \backslash F) \cap A)$.

Finally, we have established that $\mathcal{R}(x)$ is a monotone $\sigma$-complete orthoalgebra with RDP, therefore, the set of sharp elements of $\mathcal{R}(x)$ coincides with the set of central elements of $\mathcal{R}(x)$, see [Dvu4, Thm 3.2, Thm 5.11], but $\operatorname{Sh}(\mathcal{R}(x))=\mathcal{R}(x)$, so that $\mathcal{R}(x)$ is a Boolean $\sigma$-algebra in its own right.

As we have seen, the range of any observable on a monotone $\sigma$-complete orthoalgebra $E$ is always a Boolean sub-orthoalgebra of $E$ that is a sub-orthoalgebra of $E$. For example, if $E$ is an orthoalgebra, given $a \in E$, the set $\left\{0, a, a^{\prime}, 1\right\}$ is always a Boolean subalgebra of $E$, hence $E$ can be covered by a system of Boolean sub-orthoalgebras of $E$, see also [FGR, Lem 3.2]. In addition, if $E$ is a monotone $\sigma$-complete orthoalgebra, and $x$ is an observable defined by (3.0), then $E$ can be covered by a system of Boolean $\sigma$-sub-orthoalgebras of $E$.

Lemma 4.5. (1) Let $B$ and $B_{1}$ be two Boolean sub-orthoalgebras of an orthoalgebra $E$ such that $B \subseteq B_{1}$. If $a, b \in B$, then $a \vee_{B} b=a \vee_{B_{1}} b$ and $a \wedge_{B} b=a \wedge_{B_{1}} b$.

(2) Any orthoalgebra $E$ that is not a Boolean algebra can be covered by the system of maximal Boolean sub-orthoalgebras of $E$.

Proof. (1) Let $B \subseteq B_{1}$ and let $a, b \in B$. Since $B$ is in fact a sub-orthoalgebra of $E$ such that if $x+y$ exists for $x, y \in B$, then $x+y=x \vee_{B} y$. In addition, RDP holds in $B$ because $B$ is a Boolean algebra. Since $a+a^{\prime}=b+b^{\prime}$, there are four elements $c_{11}, c_{12}, c_{21}, c_{22} \in B$ such that $a=c_{11}+c_{12}, a^{\prime}=c_{21}+c_{22}, b=c_{11}+c_{21}$ and $b^{\prime}=c_{12}+c_{22}$. Then the element $c:=c_{11}+c_{12}+c_{21}$ exists in $B$ so that $c=a \vee_{B} c_{21}=b \vee_{B} c_{12}$. If $x \in B$ is an upper bound for $a, b$, then $x \geq a, b, c_{12}, c_{21}$ so that $x \geq c$ which proves $c=a \vee_{B} b$. Since the elements $c_{11}, c_{12}, c_{21}, c_{22}$ are also in $B_{1}$, we have $c \in B_{1}$ and $c=a \vee_{B_{1}} b$.

(2) Let $x \in E$ be an arbitrary element and let $\left\{B_{t}: t \in T\right\}$ be a chain of Boolean sub-orthoalgebras of $E$ containing $x$, where each $B_{t} \neq E$; such Boolean sub-orthoalgebras exist because $\left\{0, x, x^{\prime}, 1\right\}$ is such a Boolean sub-orthoalgebra. If we set $B_{0}=\bigcup_{t} B_{t}$, then $B_{0}$ is clearly a sub-orthoalgebra of $E$. If $a, b \in B_{0}$, then there is $t_{0}$ such that $a, b \in B_{t_{0}}$, and let $c=a \vee_{B_{t_{0}}} b$. We assert that $c$ is the join in the whole $B_{0}$. Indeed, let $x \in B_{0}$ be an upper bound of $a, b$. There is $t_{1}$ such that $a, b, x \in B_{t_{1}}$. By the first part of the present proof, $c=a \vee_{B_{t_{1}}} b \leq x$ and $c=a \vee_{B_{0}} b$. Hence, $B_{0}$ is a Boolean algebra and $B_{0} \neq E$ because $E$ is not a Boolean algebra. Applying Zorn's lemma, we have that there is a maximal Boolean sub-orthoalgebra $E$. Consequently, $E$ can be covered by the set of maximal Boolean sub-orthoalgebras of $E$.

Maximal Boolean sub-orthoalgebras of an orthoalgebra in which RDP holds are maximal sub-orthoalgebras with RDP and vice-versa. Indeed, every element of 
a maximal sub-orthoalgebra with RDP is central, so that, blocks are maximal Boolean sub-orthoalgebras of the orthoalgebra. In other words, maximal Boolean sub-orthoalgebras of orthoalgebras play the same role as blocks do in homogeneous effect algebras.

For example, the well-known orthoalgebra, the Fano plane, see e.g. $\mathrm{DvPu}$, p. 101], consists of seven Boolean sub-orthoalgebras with eight elements; they are only blocks.

It is worthy of recalling that if $B$ is any Boolean sub-orthoalgebra of an orthoalgebra $E$, then (i) for each $a, b \in B, a \leftrightarrow b$, (ii) if $a, b \in B, a=a_{1}+c, b=b_{1}+c$ and $a_{1}+b_{1}+c$ exists in $E$, where $a_{1}, b_{1}, c \in B$, then $a \vee_{B} b=a_{1}+b_{1}+c, a \wedge_{B} b=c$, and $a_{1} \wedge_{B} b_{1}=0$.

Lemma 4.6. (1) Let $B$ be a Boolean sub-orthoalgebra of an orthoalgebra $E$ satisfying RIP. Then any two elements $a, b \in B$ are strongly compatible, $a \vee b$ exists in $E$ and $a \vee b=a \vee_{B} b$, and $a \wedge b=a \wedge_{B} b$.

(2) If $a, b$ are arbitrary elements of any orthoalgebra $E$ with RIP such that $a+b$ exists in $E$. Then $a$ and $b$ are strongly compatible, $a \vee b$ exists, and $a \vee b=a+b$.

Proof. (1) As it was just said above, every two elements of $B$ are compatible.

(i) Assume that $a+b$ is defined in $E$. Then $a \vee_{B} b=a+b$. Since $a$ and $b$ are compatible in $B, a=a_{1}+c, b=b_{1}+c$, and $a_{1}+b_{1}+c \in B$ for some $a_{1}, b_{1}, c \in B$. Then $a_{1}+b_{1}+c=a \vee_{B} b=a+b$, so that $c=0$. Let for some $x \in E$, we have $x \leq a, b$. Then $x \leq a \leq b^{\prime}$ gives $x=0$ which implies $a_{1} \wedge b_{1}$ exists in $E$ and $a \wedge b=0$. Applying [Dvu3, Prop 2.2], we have that $a \vee b$ exists in $E$ and $a \vee b=a+b$. But $a \vee_{B} b=a+b$.

(ii) Now let $a, b \in B$ be arbitrary, then $a=a_{1}+c, b=b_{1}+c, a_{1}+b_{1}+c=a \vee_{B} b$. By the first part of the present proof, $a \vee b_{1}=a_{1}+b_{1}+c=b \vee a_{1}$ which yields $a \vee b$ exists in $E$, and $a \vee b=a_{1}+b_{1}+c=a \vee_{B} b$. Because $a$ and $b$ are arbitrary elements of $B$, then $a^{\prime} \vee b^{\prime}$ exists in $B$ so that $a \wedge b=\left(a^{\prime} \vee b^{\prime}\right)^{\prime}$ is defined in $B$. This yields that $a \wedge b$ and $a \vee b$ are defined in $B$ and they coincide with $a \wedge_{B} b$ and $a \vee_{B} b$, respectively. This in particular implies that if $a=a_{1}+c, b=b_{1}+c$ and $a_{1}+b_{1}+c$ exists in $B$ for some $a_{1}, b_{1}, c \in B$, then $a_{1} \wedge b_{1}=a_{1} \wedge_{B} b_{1}=0$ which implies $a$ and $b$ are strongly compatible.

(2) Set $a_{1}=a, a_{2}=b, a_{3}=(a+b)^{\prime}$ and define an observable $x$ via (3.0). Then $B=\mathcal{R}(x)$ is by Theorem 4.4 a Boolean sub-orthoalgebra of $E$. Hence, by (1), $a$ and $b$ are strongly compatible, so that $a \vee_{B} b=a+b=a \vee b$.

The notion of an observable was defined as a mapping $x$ from $\mathcal{B}(\mathbb{R})$ into a monotone $\sigma$-complete effect $E$. We can extend this notion also for an arbitrary effect algebra $E$ assuming that $x: \mathcal{B}(\mathbb{R}) \rightarrow E$ such that (i) $x(\mathbb{R})=1$, (ii) $x(A \cup B)=$ $x(A)+x(B)$ whenever $A, B \in \mathcal{B}(\mathbb{R})$ are disjoint, and (iii) if $\left\{A_{i}\right\} \nearrow A$, then $\bigvee_{i} x\left(A_{i}\right)$ is defined in $E$ and $x(A)=\bigvee_{i} x\left(A_{i}\right)$. A mapping $x: \mathcal{B}(\mathbb{R}) \rightarrow E$ satisfying (i) and (ii) is said to be an $f$-observable (f stands for finitely additive). It has analogous properties as do observables stated in the beginning of the third section just after definition of an observable besides (vi).

Lemma 4.7. Let $E$ be an orthoalgebra with RIP and let $a, b \in E$. The following statements are equivalent:

(i) The elements $a$ and $b$ are strongly compatible.

(ii) The elements $a$ and $b$ are compatible. 
(iii) There is an $f$-observable $x$ of $E$ and two Borel sets $A$ and $B$ of $\mathbb{R}$ such that $a=x(A)$ and $b=x(B)$.

(iv) There is a Boolean sub-orthoalgebra of $E$ containing $a$ and $b$.

Proof. Evidently (i) implies (ii).

(ii) $\Rightarrow$ (iii). There are elements $a_{1}, b_{1}, c \in E$ such that $a=a_{1}+c, b=b_{1}+c$, and $a_{1}+b_{1}+c$ is defined in $E$. Take summable elements $a_{1}, b_{1}, c,\left(a_{1}+b_{1}+c\right)^{\prime}$ and define an f-observable $x$ via (3.0). Then $a, b \in \mathcal{R}(x)$.

(iii) $\Rightarrow$ (iv). This follows easily from Theorem 4.4

(iv) $\Rightarrow$ (i). Let $B$ be a Boolean sub-orthoalgebra of $E$ containing $a$ and $b$. By Lemma 4.6, $a$ and $b$ are strongly compatible.

Lemma 4.8. Let $a$ be an element of an orthoalgebra $E$ satisfying RIP, and let $B(a)=\{b \in E: b \stackrel{\mathrm{c}}{\longleftrightarrow} a\}$. Then $B(a)$ is a sub-orthoalgebra of $E$ that is an orthomodular poset.

If, in addition, $E$ is monotone $\sigma$-complete, then $B(a)$ is also monotone $\sigma$ complete.

Every orthoalgebra with RIP is an orthomodular poset.

Proof. It is clear that $0,1, a \in B(a)$.

We show that if $b \in B(a)$, then $b^{\prime} \in B(a)$. This follows from the criterion (ii) of Theorem 4.7.

Assume $b_{1}, b_{2} \in B(a)$ and let $b_{1}+b_{2}$ exist in $E$. By Lemma 4.6(2), $b_{1}$ and $b_{2}$ are strongly compatible, and $b_{1} \vee b_{2}=b_{1}+b_{2}$. Applying [Dvu3, Prop 2.9], $a$ is strongly compatible with $b_{1} \vee b_{2}=b_{1}+b_{2}$.

To show that $B(a)$ is an OMP in its own right, we use the criterion FGR, Thm 2.12] (see also $\mathrm{DvPu}$, Prop 1.5.6]) saying that an orthoalgebra is an OMP iff the existence of $b+c$ implies $b \vee a$ exists. Thus let $b, c \in B(a)$ and let $b+c \in E$. As it was already said, Lemma 4.6(2), $b, c$ are strongly compatible, so that $b \vee c$ exists in $B(a)$, and $b \vee c=b+c$.

Let $E$ be monotone $\sigma$-complete and let $\left\{b_{n}\right\}$ be a non-decreasing sequence of elements of $B(a)$, and let $b=\bigvee_{n} b_{n}$. By [Dvu5, Prop 3.2], $b \stackrel{\mathrm{c}}{\longleftrightarrow} a$, proving $b \in B(a)$.

To establish that $E$ is an OMP, we use the fact that if we set $a=0$, then $B(a)=E$, and therefore, as it was just proved, $E$ is an OMP.

We remark that an effect algebra with RIP is not necessarily an orthomodular poset. Indeed, if an effect algebra $E$ has RDP (so it has RIP) and by $\overline{\operatorname{Rav}}, E$ is isomorphic to an interval effect algebra $\Gamma(G, u)$ and these effect algebras are not orthomodular posets, in general.

The following result follows directly from Lemma 4.8 ,

Theorem 4.9. Every monotone $\sigma$-complete orthoalgebra with RIP satisfies OEP.

Proof. Let $E$ be a monotone $\sigma$-complete orthoalgebra with RIP. By Lemma 4.8, $E$ is a monotone $\sigma$-complete orthomodular poset, equivalently, $E$ is a $\sigma$-orthocomplete orthomodular poset. In view of [DvKu, Thm 3.8], $E$ has OEP.

We note that that there are OMPs where RIP fails. Indeed, let $\Omega=\{1, \ldots, 8\}$ and let $E$ be the system of all subsets of $\Omega$ with even number of elements. Then $E$ is an OMP and since $\{1,2\},\{1,3\} \subseteq\{1,2,3,4\},\{1,2,3,5\}$, RIP fails. On the other 
side, every finite OMP with RIP is a lattice, and there are also infinitely-countable OMP's with RIP that are not lattices, see [DvPt, Thm 2.3(ii)].

The question whether every monotone $\sigma$-complete orthoalgebra belongs to the class $\mathcal{O E P}(E A)$ is still open. If each block of a monotone $\sigma$-complete orthoalgebra would be a monotone $\sigma$-complete sub-orthoalgebra, then the answer to the first question would be positive. Unfortunately, also the answer to the second question is unknown, see also [Sch, Sec 3, p. 3311].

\section{Conclusion}

The Riesz Decomposition Property is an important notion that allows us to show that effect algebras with RDP are in fact interval ones in some unital Abelian pogroups with interpolation. A more general notion are homogeneous effect algebras.

Observables model quantum mechanical measurements. They are defined as $\sigma$-homomorphisms from the set of Borel sets on $\mathbb{R}$ preserving order, negations, addition, and monotone limits. We have studied a problem when a given system of elements, called a spectral resolution, of a monotone $\sigma$-complete effect algebra implies the existence of the corresponding observable. Theorem 3.3 says that every representable monotone $\sigma$-complete effect algebra has this property, and Theorem 4.9 describes an analogous result for monotone $\sigma$-complete effect algebras with the Riesz Interpolation Property.

In Theorem 4.1 we showed that the set of sharp elements of a homogeneous monotone $\sigma$-complete effect algebra is always a monotone $\sigma$-complete sub-effect algebra. This result was applied in Proposition 4.3 to sharp observables showing their characterization by the corresponding spectral resolution whose elements are only sharp elements. In addition, in Lemma 4.8 we showed that every orthoalgebra with RIP is in fact an OMP.

\section{REFERENCES}

[Bau] H. Bauer, "Probability Theory", de Gruyter, Berlin 1996.

[BiNe] G. Birkhoff, J. von Neumann, The logic of quantum mechanics, Ann. Math. 37 (1936), 823-834.

[BCD] D. Buhagiar, E. Chetcuti, A. Dvurečenskij, Loomis-Sikorski representation of monotone б-complete effect algebras, Fuzzy Sets and Systems 157 (2006), 683-690.

[Dvu1] A. Dvurečenskij, Measures and $\perp$-decomposable measures on effects of a Hilbert space, Atti Sem. Mat. Fis. Univ. Modena 45 (1997), 259-288.

[Dvu2] A. Dvurečenskij, Loomis-Sikorski theorem for $\sigma$-complete $M V$-algebras and $\ell$-groups, J. Austral. Math. Soc. Ser. A 68 (2000), 261-277.

[Dvu3] A. Dvurečenskij, On effect algebras which can be covered by $M V$-algebras, Inter. J. Theor. Phys. 41 (2002), 221-229.

[Dvu4] A. Dvurečenskij, Central elements and Cantor-Bernstein's theorem for pseudo-effect algebras, J. Austral. Math. Soc. 74 (2003), 121-143.

[Dvu5] A. Dvurečenskij, Representable effect algebras and observables, Inter. J. Theor. Phys. 53 (2014), 2855-2866. DOI: 10.1007/s10773-014-2083-z

[Dvu6] A. Dvurečenskij, Olson order of quantum observables, Inter. J. Theor. Phys. DOI:10.1007/s10773-016-3113

[DvKu] A. Dvurečenskij, M. Kuková, Observables on quantum structures, Inf. Sci. 262 (2014), 215-222. DOI: 10.1016/j.ins.2013.09.014

[DvPt] A. Dvurečenskij, P. Pták, Quantum logics with the Riesz interpolation property, Math. Nachr. 271 (2004), 10-14.

[DvPu] A. Dvurečenskij, S. Pulmannová, "New Trends in Quantum Structures”, Kluwer Academic Publ., Dordrecht, Ister Science, Bratislava, 2000, $541+$ xvi pp. 
[FoBe] D.J. Foulis, M.K. Bennett, Effect algebras and unsharp quantum logics, Found. Phys. 24 (1994), 1325-1346.

[FGR] D.J. Foulis, R.J. Greechie, G.T. Rüttimann, Filters and supports in orthoalgebras, Inter. J. Theor. Phys. 31 (1992), 789-807.

[Go] K.R. Goodearl, "Partially Ordered Abelian Groups with Interpolation", Math. Surveys and Monographs No. 20, Amer. Math. Soc., Providence, Rhode Island, 1986.

[Gud] S.P. Gudder, Uniqueness and existence properties of bounded observables, Pacific J. Math. 19 (1966), 81-93.

[Hal] P.R. Halmos, "Measure Theory", Springer-Verlag, Berlin, 1974.

[Hol] A.S. Holevo, "Probabilistic and Statistical Aspects of Quantum Theory", North-Holland, Amsterdam 1982.

[Jen] G. Jenča, Blocks of homogeneous effect algebras, Bull. Austral. Math. Soc. 64 (2001), 81-98.

[JeRi] G. Jenča, Z. Riečanová, On sharp elements in lattice ordered effect algebras, Busefal $\mathbf{8 0}$ (1999), 24-29.

[JPV1] A. Jenčová, S. Pulmannová, E. Vinceková, Sharp and fuzzy observables on effect algebras, Inter. J. Theor. Phys. 47 (2008), 125-148.

[JPV2] A. Jenčová, S. Pulmannová, E. Vinceková, Observables on $\sigma$-MV-algebras and $\sigma$-lattice effect algebras, Kybernetika 47 (2011), 541-559.

[Kal] O. Kallenberg, "Foundations of Modern Probability", Springer-Verlag, New York, Berlin, Heidelberg, 1997.

[Mun] D. Mundici, Tensor products and the Loomis-Sikorski theorem for MV-algebras, Advan. Appl. Math. 22 (1999), 227-248.

[Ols] M.P. Olson, The self-adjoint operators of a von Neumann algebra form a conditionally complete lattice, Proc. Amer. Math. Soc. 28 (1971), 537-544.

[Rav] K. Ravindran, On a structure theory of effect algebras, PhD thesis, Kansas State Univ., Manhattan, Kansas, 1996.

[Sch] B.S.W. Shröder, On three notions of orthosummability in orthoalgebras, Inter. J. Theor. Phys. 38 (1999), 3305-3313.

[Var] V.S. Varadarajan, "Geometry of Quantum Theory", Vol. 1, van Nostrand, Princeton, New Jersey, 1968. 